\title{
Constraints on a scalar-tensor model with Gauss-Bonnet coupling from SN Ia and BAO observations
}

\author{
S. Bellucci $^{a}$ 1, A. Banijamali ${ }^{b}{ }^{2}$, B. Fazlpour ${ }^{c}{ }^{3}$ and M. Solbi ${ }^{b}$ \\ ${ }^{a}$ INFN - Laboratori Nazionali di Frascati, 1-00044, Frascati (Rome), Italy \\ ${ }^{b}$ Department of Basic Sciences, Babol Noshirvani University of Technology, Babol, Iran \\ ${ }^{c}$ Department of Physics, Babol Branch, Islamic Azad University, Babol, Iran
}

\begin{abstract}
In the present work, the observational consequences of a subclass of of the Horndeski theory have been investigated. In this theory a scalar field (tachyon field) is non-minimally coupled to the Gauss-Bonnet invariant through an arbitrary function of the scalar field. By considering a spatially flat FRW universe, the free parameters of the model have been constrained using a joint analysis from observational data of the Type Ia supernovae and Baryon Acoustic Oscillations measurements. The best fit values obtained from these datasets are then used to reconstruct the equation of state parameter of the scalar field. The results show the phantom, quintessence and phantom divide line crossing behavior of the equation of state and also cosmological viability of the model.
\end{abstract}

PACS numbers: 95.36.+x, 98.80.-k, 04.50.kd

Keywords: Dark energy; Gauss-Bonnet coupling; Observational cosmology

\footnotetext{
${ }^{1}$ Stefano.Bellucci@lnf.infn.it

2a.banijamali@nit.ac.ir

3 b.fazlpour@umz.ac.ir
} 


\section{Introduction}

The current accelerated expansion of the universe is one of the great problems of modern cosmology. This acceleration was first suggested by Type Ia supernovae (SN Ia) surveys [1, 2] and then by measurements of the cosmic microwave background (CMB) [3, 4, the Hubble constant [5], Baryon Acoustic Oscillations (BAO) [6] and more measurements of Type Ia supernovae [7. Although observational cosmology confirms the acceleration of the universe, explaining this issue from theoretical point of view is a big challenge. The simplest way to obtain an accelerated universe is adding a cosmological constant to the standard cosmological model. However, a cosmological constant suffers from the fine-tuning problem, that is due to its extremely small observed value compared to predictions from theoretical considerations [8. As a result one can follow two ways to explain the late-time behavior of the universe: modifying general relativity at large scale [9] or introducing a new content in the universe such as canonical scalar field, phantom scalar, both scalars, vector fields etc., that is introducing the concept of dark energy [10, 11, 12.

Furthermore, dynamical dark energy models can be extended in a huge class of models. Among them, non-minimally coupled dark energy models in which scalar fields coupled to the curvature terms dubbed scalar-tensor theories have been extensively studied in the literature. The most famous example of such theories is known as the Brans-Dicke 13 theory in which the gravitational constant is replaced by a scalar field $\phi$ entering into the action as $\phi^{2} R, R$ is the Ricci scalar. Another well-known example of non-minimally coupled system is provided by $\left(1-\xi \phi^{2}\right) R$ coupling in which $\xi$ is a constant measuring the strength of nonminimal coupling 14 .

Moreover, due to the novel features of non-minimally coupled scalar field system, such as allowing the phantom divide crossing and having the cosmological scaling solutions, these models are of great interest to the community [15, 16, 17, 18, 19, 20]. On the other hand, in 1974 Horndeski 21] found the most general class of scalar-tensor theories which lead to the second order differential equations similar to the Einstein general relativity. The Horndeski gravity has been considered in many papers in the context of the inflationary cosmology [22, 23]. An interesting subclass of the Horndeski theory is given by the non-minimal coupling of the scalar field to the Gauss-Bonnet invariant in four dimensions [34, 35, 36, 37, 38, 39, 40. Such a non-minimal coupling originates from the string theory and the trace anomaly and may play an important role in cosmological context. For example, this coupling has been proposed to address the dark energy problem in 39] and various aspects of accelerating cosmologies with Gauss-Bonnet correction have been discussed in [1, 42, 43]. Indeed, these studies yield the result that the scalar-curvature coupling predicted by fundamental theories may become important at current, low-curvature universe. It deserves mention that the modifications of gravity from the Gauss-Bonnet invariant have been often considered as the result of quantum gravity effects [4, 45, 46.

In the present work, we will consider a model in which the scalar field playing the role of dark energy is coupled to the Gauss-Bonnet invariant. Here we derive constraints on the model parameters from a combination of available SN Ia data, as well as available BAO data and $\chi^{2}$ minimization technique.

The outline of the paper is as follows: In the next section we present the basic formalism of our model in a flat FRW background, along with the definition of different cosmological parameters. We then discuss the observational dataset and methodology in section III. Our main results in data analysis are summarized in section IV. Finally, section V is devoted to our conclusions.

\section{The model and cosmological background}

The model we examine in this paper is described by the following action:

$$
S=\int d^{4} x \sqrt{-g}\left[\frac{R}{2 \kappa^{2}}-V(\varphi) \sqrt{1-\partial_{\mu} \varphi \partial^{\mu} \varphi}-\eta(\varphi) \mathcal{G}+\mathcal{L}_{m}\right],
$$

where $g$ is the determinant of the metric tensor, $\kappa^{2}=8 \pi G, G$ is the gravitational constant and $\mathcal{L}_{m}$ is the matter Lagrangian density. The second term in the brackets is the Lagrangian of tachyon field with the potential $V(\phi)$, while the third term represents a non-minimal coupling between the scalar field and 
curvature through a general function $\eta(\phi) . \mathcal{G}$ is the Gauss-Bonnet invariant which is given by:

$$
\mathcal{G}=R^{2}-4 R_{\mu \nu} R^{\mu \nu}+R_{\mu \nu \lambda \rho} R^{\mu \nu \lambda \rho}
$$

where $R, R_{\mu \nu}, R_{\mu \nu \lambda \rho}$ are the Ricci scalar, the Ricci tensor and the Riemann tensor, respectively.

Notice that not only tachyon field originates from the string theory but also the term proportional to the Gauss-Bonnet invariant $\mathcal{G}$ is considered as a stringy correction in the action. These are our main motivations to study the model.

To analyse the model it is more convenient to use the following redefinition, as proposed in 47] for studying the tachyon dynamics,

$$
\varphi \rightarrow \phi=\int d \varphi \sqrt{V(\varphi)} \Longleftrightarrow \partial \varphi=\frac{\partial \phi}{\sqrt{V(\phi)}}
$$

Applying (3) in (1) yields to our starting action as follows:

$$
S=\int d^{4} x \sqrt{-g}\left[\frac{R}{2 \kappa^{2}}-V(\phi) \sqrt{1-\frac{\partial_{\mu} \phi \partial^{\mu} \phi}{V(\phi)}}-\eta(\phi) \mathcal{G}+\mathcal{L}_{m}\right] .
$$

The variation of the action (4) with respect to the metric leads to the following gravitational equations:

$$
R_{\mu \nu}-\frac{1}{2} g_{\mu \nu} R=\kappa^{2}\left(T_{\mu \nu}^{\phi}+T_{\mu \nu}^{G B}+T_{\mu \nu}^{m}\right),
$$

where $T_{\mu \nu}^{m}$ is the usual energy-momentum tensor for the matter, $T_{\mu \nu}^{\phi}$ corresponds to the energy-momentum tensor of minimally coupled tachyon scalar field and $T_{\mu \nu}^{G B}$ is the contribution of the non-minimal GaussBonnet coupling. These last two components are given by

$$
T_{\mu \nu}^{\phi}=-u \nabla_{\mu} \phi \nabla_{\nu} \phi-g_{\mu \nu} u^{-1} V(\phi)
$$

and

$$
\begin{aligned}
T_{\mu \nu}^{G B}= & 4\left(\left[\nabla_{\mu} \nabla_{\nu} \eta(\phi)\right] R-g_{\mu \nu}\left[\nabla_{\rho} \nabla^{\rho} \eta(\phi)\right] R-2\left[\nabla^{\rho} \nabla_{\mu} \eta(\phi)\right] R_{\nu \rho}-2\left[\nabla^{\rho} \nabla_{\nu} \eta(\phi)\right] R_{\mu \rho}\right. \\
& \left.+2\left[\nabla_{\rho} \nabla^{\rho} \eta(\phi)\right] R_{\mu \nu}+2 g_{\mu \nu}\left[\nabla^{\rho} \nabla^{\sigma} \eta(\phi)\right] R_{\rho \sigma}-2\left[\nabla^{\rho} \nabla^{\sigma} \eta(\phi)\right] R_{\mu \rho \nu \sigma}\right),
\end{aligned}
$$

where $u=\sqrt{1-\frac{\partial_{\mu} \phi \partial^{\mu} \phi}{V(\phi)}}$.

In the derivation of $T_{\mu \nu}^{G B}$, the properties of the 4-dimensional Gauss-Bonnet invariant have been used (see [39, 48, for details). The energy density and pressure derived from these energy-momentum tensors will be considered as effective ones and we represent them by $\rho_{D E}$ and $p_{D E}$, respectively.

Now, we assume the spatially flat Friedmann-Robertson-Walker (FRW) metric,

$$
d s^{2}=-d t^{2}+a^{2}(t)\left(d r^{2}+r^{2} d \Omega^{2}\right),
$$

where $a(t)$ is the scale factor. Considering this metric in equations (5)-(7) we obtain the following Friedmann equations:

$$
\begin{gathered}
H^{2}=\frac{\kappa^{2}}{3}\left(\rho_{D E}+\rho_{m}\right), \\
\dot{H}=-\frac{\kappa^{2}}{2}\left(\rho_{D E}+p_{D E}+\rho_{m}+p_{m}\right),
\end{gathered}
$$

where $\rho_{m}$ and $p_{m}$ are the energy density and pressure of the matter, $\rho_{D E}$ and $p_{D E}$ are given by

$$
\rho_{D E}=u V(\phi)+24 H^{3} f(\phi) \dot{\phi}
$$


and

$$
p_{D E}=-u^{-1} V(\phi)-8 H^{2}\left(f_{, \phi} \dot{\phi}^{2}+f(\phi) \ddot{\phi}\right)-16 H f(\phi) \dot{\phi}\left(\dot{H}+H^{2}\right),
$$

where $H=\frac{\dot{a}}{a}$ is the Hubble parameter, and we have also defined $f(\phi)=\frac{d \eta}{d \phi}, f_{, \phi}=\frac{d f(\phi)}{d \phi}$.

Further, by varying the action (4) over $\phi$ and assuming that $\phi$ only depends on time, we obtain the equation of motion for $\phi$, which in FRW background takes the following form

$$
\ddot{\phi}+3 u^{-2} H \dot{\phi}+\left(1-\frac{3 \dot{\phi}^{2}}{2 V}\right) V_{, \phi}+24 H^{2}\left(\dot{H}+H^{2}\right) f(\phi)=0 .
$$

Note that in deriving equation (13), we have used the following expression for the Gauss-Bonnet invariant in FRW background

$$
\mathcal{G}=24 H^{2}\left(\dot{H}+H^{2}\right) \text {. }
$$

In addition, the energy conservation equations for dark energy and the matter are expressed in the following forms, respectively

$$
\dot{\rho}_{D E}+3 H\left(1+\omega_{D E}\right) \rho_{D E}=0
$$

and

$$
\dot{\rho}_{m}+3 H\left(1+\omega_{m}\right) \rho_{m}=0,
$$

where $\omega_{D E}=\frac{p_{D E}}{\rho_{D E}}$ and $\omega_{m}=\frac{p_{m}}{\rho_{m}}$ are the equation of state parameters of dark energy and matter respectively. Here, we just focus on the late-time eras, so that we can neglect the radiation contribution and assume a pressureless fluid for the matter content $\omega_{m}=\frac{p_{m}}{\rho_{m}}=0$. Then, the continuity equation (16) can be easily integrated to yield

$$
\rho_{m}=\rho_{m_{0}}\left(\frac{a_{0}}{a}\right)^{-3}=\rho_{m_{0}}(1+z)^{3} .
$$

where $\rho_{m_{0}}$ denotes the present value of the matter energy density and $z$ is the redshift parameter $z+1=\frac{a_{0}}{a}$. In addition, we define the density parameters of dark energy and the matter by $\Omega_{D E}=\left(\kappa^{2} \rho_{D E}\right) /\left(3 H^{2}\right)$ and $\Omega_{m}=\left(\kappa^{2} \rho_{m}\right) /\left(3 H^{2}\right)$ and here after a subscript " 0 " for a parameter stands for the present value of that parameter.

Before closing this section, it is worthwhile to mention that since we are going to constrain the model using observational data, which are expressed in terms of the redshift, it is convenient to rewrite the Friedmann equations in terms of the latter, instead of the cosmic time. This can be done straightforwardly by the following replacements in equations (11) and (12),

$$
\begin{array}{lr}
\dot{H}=-H H^{\prime}(1+z), & \dot{\phi}=-H(1+z) \phi^{\prime}, \\
\ddot{\phi}=H^{2}(1+z) \phi^{\prime}+H H^{\prime}(1+z)^{2} \phi^{\prime}+H^{2}(1+z)^{2} \phi^{\prime \prime},
\end{array}
$$

where prime denotes the derivative with respect to the redshift.

\section{Methods}

Here, we explain the methodology that we use to constrain the model by using the recent observational datasets from Type Ia Supernova (SNe Ia) and Baryon Acoustic Oscillations (BAO).

We use the Markov-chain Monte Carlo (MCMC) method for the minimization of $\chi^{2}$ to perform the statistical analysis. We have tested the model using the publicly available codes by S. Nesseris et a.l. (see for example [49, 50, ?]) and making the necessary changes in the case of our model. Now, we briefly explain the method for elaborating the observational data.

Our study follows the likelihood $\mathcal{L} \propto \exp \left(-\chi^{2} / 2\right)$, where the total $\chi^{2}$ for combined datasets reads:

$$
\chi_{\text {total }}^{2}=\chi_{\mathrm{SN}}^{2}+\chi_{\mathrm{BAO}}^{2} .
$$

In the following subsections, the way by which, one can calculate each of $\chi^{2}$ is described. 


\subsection{Type Ia Supernova (SN Ia)}

The $\chi^{2}$ function for the SNe Ia is given by [52],

$$
\chi_{\mathrm{SN}}^{2}=A-2 \mu_{0} B+\mu_{0}^{2} C,
$$

where $A, B$ and $C$ are defined by

$$
\begin{aligned}
A & =\sum_{i} \frac{\left[\mu_{\mathrm{obs}}\left(z_{i}\right)-\mu_{\mathrm{th}}\left(z_{i} ; \mu_{0}=0\right)\right]^{2}}{\sigma_{i}^{2}} \\
B & =\sum_{i} \frac{\mu_{\mathrm{obs}}\left(z_{i}\right)-\mu_{\mathrm{th}}\left(z_{i} ; \mu_{0}=0\right)}{\sigma_{i}^{2}} \\
C & =\sum_{i} \frac{1}{\sigma_{i}^{2}} .
\end{aligned}
$$

The definition of the distance modulus is

$$
\mu_{\mathrm{th}}(z) \equiv 5 \log _{10} D_{L}(z)+\mu_{0},
$$

where $\mu_{0} \equiv 42.38-5 \log _{10} h$, with $h \equiv H_{0} / 100 /\left[\mathrm{km} \mathrm{sec}^{-1} \mathrm{Mpc}^{-1}\right]$ [3] and the subscripts "th" and "obs" stand for theoretical and the observed distance modulus. Also, the quantity $\sigma_{i}$ represents the statistical uncertainly in the distance modulus.

The dimensionless luminosity distance $D_{L}$ for the flat universe is given by

$$
D_{L}(z)=(1+z) \int_{0}^{z} \frac{d z^{\prime}}{E\left(z^{\prime}\right)}
$$

where

$$
E(z)=\frac{H(z)}{H_{0}}=\sqrt{\Omega_{\mathrm{m}}^{(0)}(1+z)^{3}+\Omega_{\mathrm{r}}^{(0)}(1+z)^{4}+\Omega_{\mathrm{DE}}^{(0)}(1+z)^{3\left(1+w_{\mathrm{DE}}\right)}} .
$$

Here, $\Omega_{\mathrm{r}}$ is the radiation density parameter and $\Omega_{\mathrm{r}}^{(0)}=\Omega_{\gamma}^{(0)}\left(1+0.2271 N_{\text {eff }}\right)$, where $\Omega_{\gamma}^{(0)}$ is the present fractional photon energy density and $N_{\text {eff }}=3.04$ is the effective number of neutrino species 3 .

Now, the minimizing of $\chi_{\mathrm{SN}}^{2}$ with respect to $\mu_{0}$ yields to

$$
\tilde{\chi}_{\mathrm{SN}}^{2}=A-\frac{B^{2}}{C} .
$$

In our statistical analysis we use (25) for SNe Ia dataset and the Union 2.1 compilation data [51] of 580 data points have been used to constrain the model parameters.

\subsection{Baryon Acoustic Oscillations (BAO)}

Next, we have used BAO measurement dataset to put the BAO constraints on the model parameters. The BAO observable is the distance ratio $d_{z} \equiv r_{s}\left(z_{\mathrm{d}}\right) / D_{V}(z)$, where $r_{s}$ is the comoving sound horizon, $z_{\mathrm{d}}$ is the redshift at the drag epoch [53] and $D_{V}$ is the volume-averaged distance which is defined as follows [54,

$$
D_{V}(z) \equiv\left[(1+z)^{2} D_{A}^{2}(z) \frac{z}{H(z)}\right]^{1 / 3}
$$

In equation (26) $D_{A}(z)$ is the proper angular diameter distance for the flat universe.

Here we have considered six BAO data points (see Table 1). The WiggleZ collaboration [55] has measured the baryon acoustic scale at three different redshifts, while SDSS and 6DFGS surveys provide data at lower 
redshift [53].

\begin{tabular}{|c|c|cc|ccc|}
\multicolumn{1}{c}{$6 \mathrm{dF}$} & \multicolumn{2}{c}{ SDSS } & \multicolumn{3}{c|}{ WiggleZ } \\
\hline$z$ & 0.106 & 0.2 & 0.35 & 0.44 & 0.6 & 0.73 \\
\hline$d_{z}$ & 0.336 & 0.1905 & 0.1097 & 0.0916 & 0.0726 & 0.0592 \\
\hline$\Delta d_{z}$ & 0.015 & 0.0061 & 0.0036 & 0.0071 & 0.0034 & 0.0032 \\
\hline
\end{tabular}

Table 1: The BAO data used in our analysis.

The $\chi^{2}$ function of the BAO data is defined as,

$$
\chi_{\mathrm{BAO}}^{2}=\left(x_{i, \mathrm{BAO}}^{\mathrm{th}}-x_{i, \mathrm{BAO}}^{\mathrm{obs}}\right)\left(C_{\mathrm{BAO}}^{-1}\right)_{i j}\left(x_{j, \mathrm{BAO}}^{\mathrm{th}}-x_{j, \mathrm{BAO}}^{\mathrm{obs}}\right),
$$

where the indices $i, j$ are in growing order in $z$, as in Table 1 and $C_{\mathrm{BAO}}^{-1}$ can be obtained by the covariance data [55] in terms of $d_{z}$ as follows:

$$
C_{\mathrm{BAO}}^{-1}=\left(\begin{array}{cccccc}
4444 & 0 & 0 & 0 & 0 & 0 \\
0 & 30318 & -17312 & 0 & 0 & 0 \\
0 & -17312 & 87046 & 0 & 0 & 0 \\
0 & 0 & 0 & 23857 & -22747 & 10586 \\
0 & 0 & 0 & -22747 & 128729 & -59907 \\
0 & 0 & 0 & 10586 & -59907 & 125536
\end{array}\right) .
$$

One can now obtain the best fit values of the model parameters by minimizing $\chi_{\text {total }}^{2}$ in equation (19).

\section{Observational constraints on the model parameters}

Following the $\chi^{2}$ analysis (as presented in the previous section), in this section, we obtain the constraints on the free parameters of the model.

Two important functions in our analysis are the function $f(\phi)$ and the scalar field potential $V(\phi)$. We will consider power-law and exponential forms for $f(\phi)$ and $V(\phi)$ and thus our study is categorized into the following four different cases:

Case I: Exponential $f(\phi)$ and Power-law $V(\phi)$

$$
f(\phi) \propto e^{\alpha \phi}, V(\phi) \propto \phi^{\beta}
$$

Case II: Power-law $f(\phi)$ and Exponential $V(\phi)$

$$
f(\phi) \propto \phi^{\alpha}, V(\phi) \propto e^{\beta \phi}
$$

Case III: Power-law $f(\phi)$ and $V(\phi)$

$$
f(\phi) \propto \phi^{\alpha}, V(\phi) \propto \phi^{\beta}
$$

Case IV: Exponential $f(\phi)$ and $V(\phi)$

$$
f(\phi) \propto e^{\alpha \phi}, V(\phi) \propto e^{\beta \phi},
$$

where $\alpha$ and $\beta$ are the free parameters which will be constrained using the data.

In the present work, we identify the parameters of the model as the parameters $\alpha$ and $\beta$, the present matter density parameter $\Omega_{m_{0}}$ and dark energy equation of state parameter $\omega_{D E}$. Thus, the model parameters are $\left(\alpha, \beta, \Omega_{m_{0}}, \omega_{D E}\right)$.

Now, performing the combined $(S N I a+B A O)$ analysis using MCMC method for the cases I-IV, yields to the constraint results as what summarized in Table 2. 


\begin{tabular}{|c|c|c|c|c|c|}
\hline model & $\chi_{\min }^{2}$ & $\Omega_{m_{0}}$ & $\omega_{D E}$ & $\alpha$ & $\beta$ \\
\hline Case I & 594.216 & $0.26 \pm 0.006$ & $-1.099 \pm 0.002$ & $-0.995 \pm 0.012$ & $0.023 \pm 0.011$ \\
\hline Case II & 663.505 & $0.26 \pm 0.003$ & $-1.199 \pm 0.003$ & $-2.998 \pm 0.025$ & $0.077 \pm 0.013$ \\
\hline Case III & 601.833 & $0.26 \pm 0.005$ & $-1.199 \pm 0.004$ & $-1.998 \pm 0.025$ & $0.23 \pm 0.016$ \\
\hline Case IV & 595.747 & $0.26 \pm 0.028$ & $-1.25 \pm 0.06$ & $-1.96 \pm 0.25$ & $0.43 \pm 0.081$ \\
\hline
\end{tabular}

Table 2: The value of $\chi_{\min }^{2}$ and the best fit values of the model parameters $\Omega_{m_{0}}, \omega_{D E}, \alpha$ and $\beta$ for the cases I-IV.

In this table, the reader may see a compact presentation of the best fit values of the model parameters as well as $\chi_{\min }^{2}$ for each case, separately.

Furthermore, in Figures $1-4$ we present the $1 \sigma, 2 \sigma$ and $3 \sigma$ confidence level contour plots for several combinations of the model parameters as well as their likelihood analysis for the cases I-IV, respectively. Additionally, in figure 5 using the same combined analysis $\mathrm{SN}$ Ia $+\mathrm{BAO}$, we have shown the qualitative evolution of the dark energy equation of state parameter. Figure 6 shows the Hubble diagram for 580 SN Ia from (Union 2.1) sample. The curves represent the distance modulus predicted by the four cases I-IV in our model.

Further, the case with the lowest value of $\chi_{\min }^{2}$ is the case I and as it is clear from Table 2 the cases with the exponential coupling function $f(\phi)$ (cases I \& IV) have a lower $\chi_{\min }^{2}$ than the cases with power-law $f(\phi)$ (cases II \& III).

The joint analysis on cases I-IV shows that the best fit values of the dark energy equation of state parameter, exhibit phantom behavior, although very close to the cosmological constant boundary. As one see from Table 2 in case I, we have the nearest value of the equation of state parameter to the cosmological constant $\left(\omega_{\Lambda}=-1\right)$ and in case IV, the phantom character of the current dark energy equation of state is the clearest one. Notice that the quintessence behavior of $\omega_{D E}$ is excluded in all cases of Table 2. Further, from Figure 5. it is clear that the transition from quintessence phase $\left(\omega_{D E}>-1\right)$ to the phantom phase $\left(\omega_{D E}<-1\right)$ or the so-called phantom divide line crossing, occurs in all four cases, which is in agreement with observational results [56, 57, 58.

From the constraints on $\alpha$ and $\beta$ as shown in Table 2, it is clear that the combination of SN Ia + BAO data favors negative values for $\alpha$ and positive values for $\beta$ in cases I-IV.

Between the best-fit values of $\alpha$, the case II i.e. when the coupling function is in power-law form, has the minimum value, while the case I in which $f(\phi)$ is exponential has the maximum value of $\alpha$. On the other hand, the best-fit value of the free parameter $\beta$, has its minimum and maximum values for the cases I and IV, that is for power-law and exponential potentials, respectively.

It deserves mention here that the values of the dark matter density parameter at present $\Omega_{m_{0}}$ for all four cases are very close to the desired value in cosmology. 


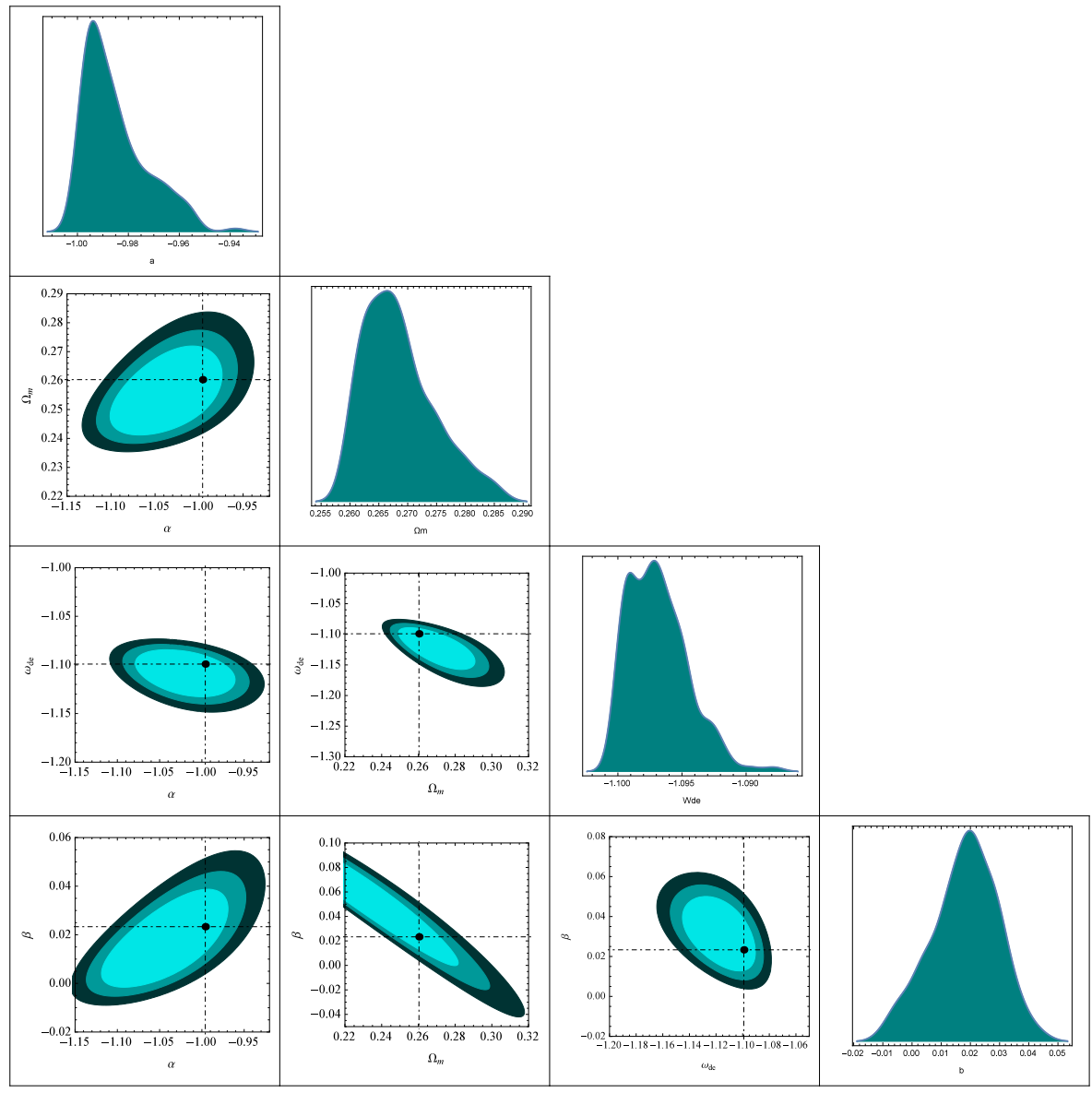

Figure 1: $1 \sigma(68.3 \%), 2 \sigma(95.4 \%)$ ans $3 \sigma(99.7 \%)$ confidence level contour plots for different combinations of the model parameters with also 1-dimensional posterior distributions in the case I for combined observational dataset from SN Ia + BAO. The black dot in each contour plot represents the best fit values of the corresponding pair. 


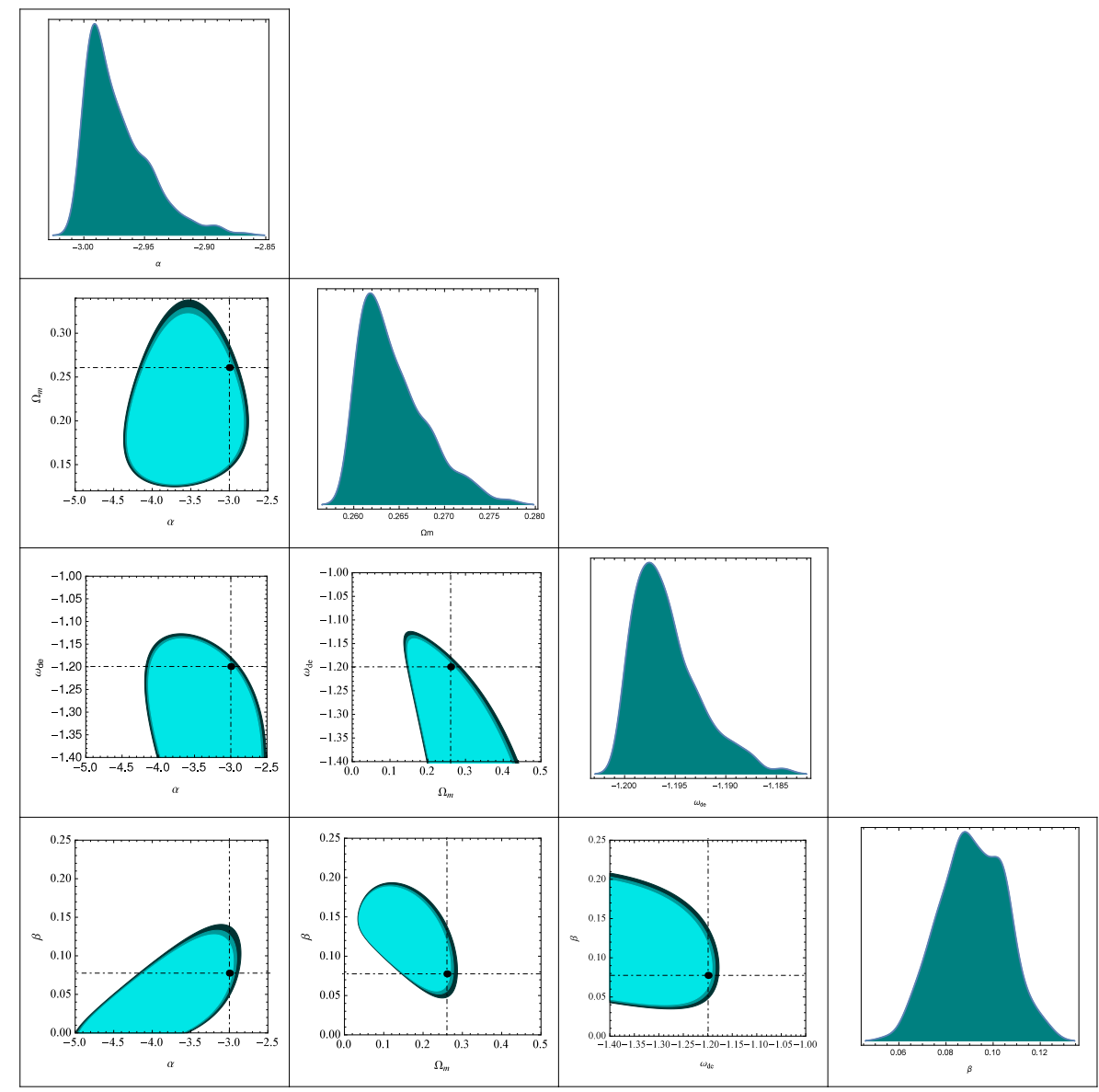

Figure 2: $1 \sigma(68.3 \%), 2 \sigma(95.4 \%)$ ans $3 \sigma(99.7 \%)$ confidence level contour plots for different combinations of the model parameters with also 1-dimensional posterior distributions in the case II for combined observational dataset from SN Ia + BAO. The black dot in each contour plot represents the best fit values of the corresponding pair. 


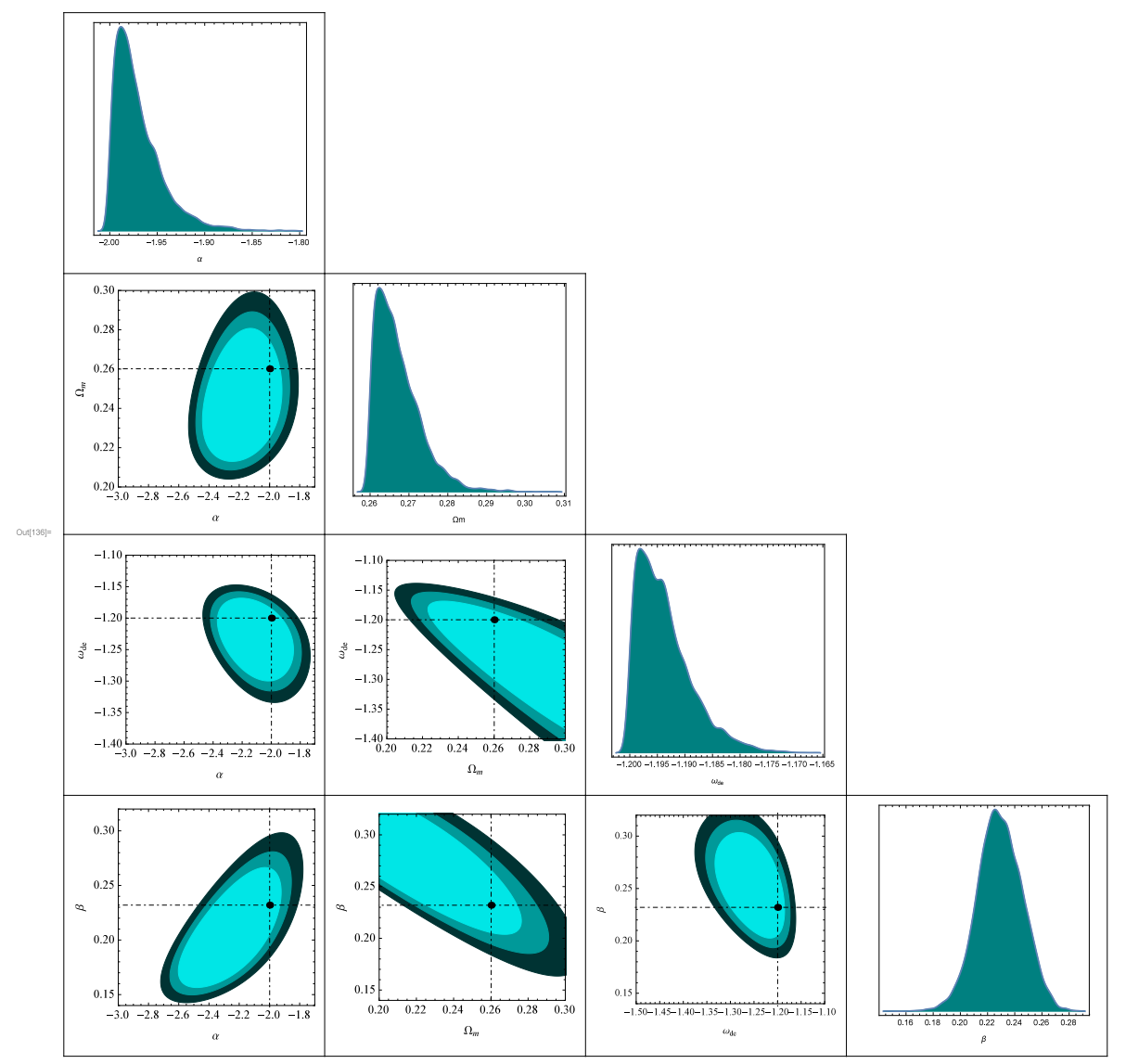

Figure 3: $1 \sigma(68.3 \%), 2 \sigma(95.4 \%)$ ans $3 \sigma(99.7 \%)$ confidence level contour plots for different combinations of the model parameters with also 1-dimensional posterior distributions in the case III for combined observational dataset from SN Ia + BAO. The black dot in each contour plot represents the best fit values of the corresponding pair. 


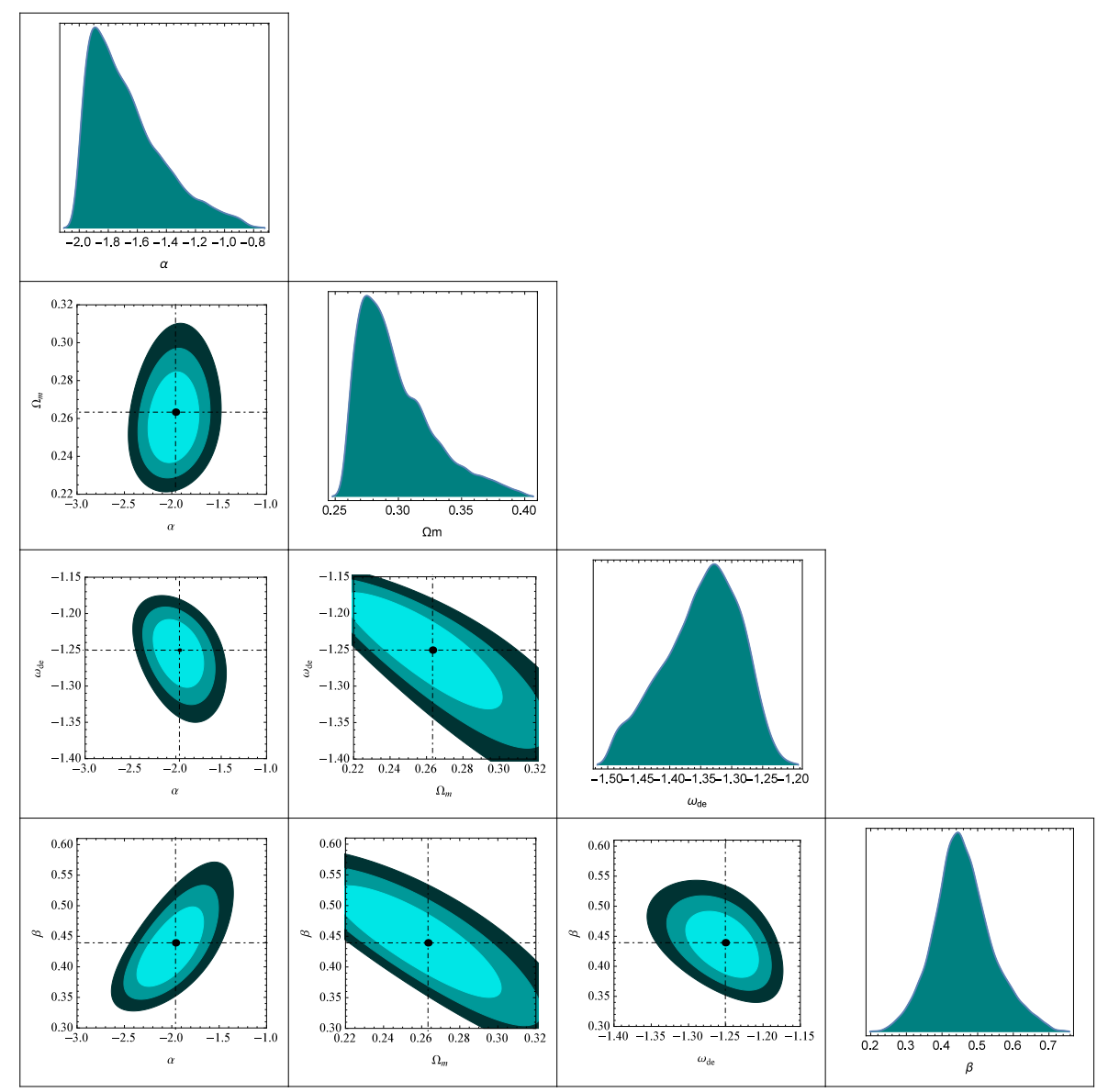

Figure $4: 1 \sigma(68.3 \%), 2 \sigma(95.4 \%)$ ans $3 \sigma(99.7 \%)$ confidence level contour plots for different combinations of the model parameters with also 1-dimensional posterior distributions in the case IV for combined observational dataset from SN Ia + BAO. The black dot in each contour plot represents the best fit values of the corresponding pair. 


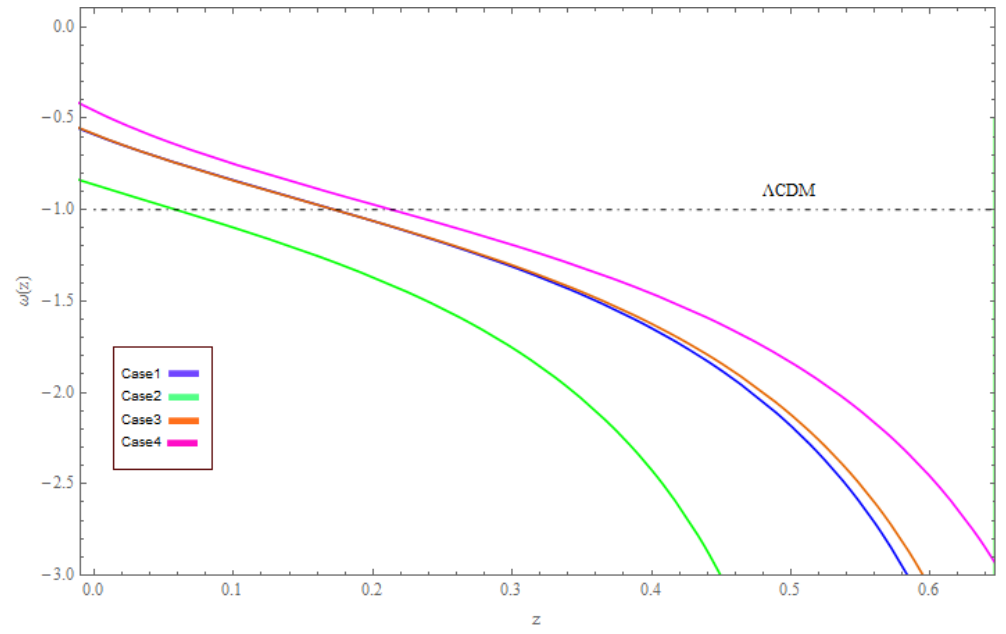

Figure 5: The evolution of the dark energy equation of state parameter, for the best fit values of $(\alpha, \beta)$ that arises from the analysis of SN Ia + BAO datasets, for the cases I (blue), II(green), III (orange) and IV (pink).

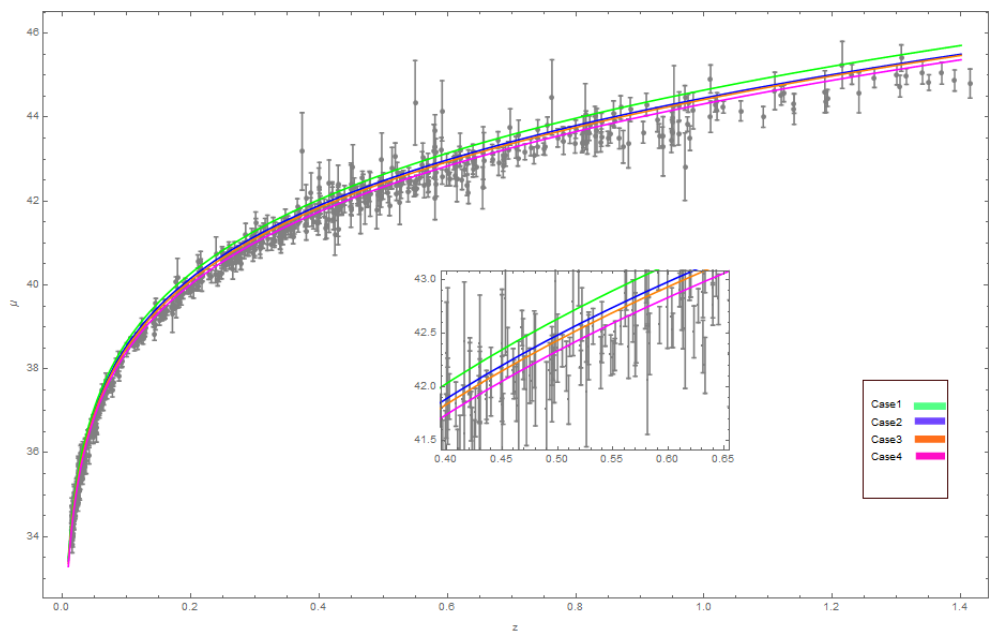

Figure 6: The Hubble diagram for 580 data of SN Ia from Union 2.1 sample 51. The curves correspond to the distance modulus predicted by the four cases I-IV with the best-fit values coming from the joint analysis of SN Ia + BAO as presented in Table 2 .

\section{Conclusion}

In this paper, we have focused on the analysis of a non-minimally coupled scalar field theory in which the scalar field is considered as a candidate of dark energy. In this model a tachyon field is non-minimally coupled to the Gauss-Bonnet invariant, via a general coupling function $f(\phi)$, as in action (4). The cosmological evolution of the model is studied by assuming a flat FRW universe. Then, we placed constraints on the free parameters of the model by performing a joint statistical analysis using the recent cosmological data from $\mathrm{SN}$ Ia and BAO measurements. We have considered the exponential and power-law forms for the scalar field potential, as well as the non-minimal coupling function. Then, we have obtained the best fit values of the free parameters $\alpha$ and $\beta$ in the potential and the coupling function, respectively. The equation of state 
parameter of dark energy, $\omega_{D E}$ and the present value of the matter density $\Omega_{m_{0}}$ have also been fitted.

According to the contents of Table 2, where our results are summarized, the joint analysis of $\mathrm{SN}$ Ia $+\mathrm{BAO}$,

favors the negative values for $\alpha$ and positive values for $\beta$. In addition, by constraining with the datasets of SN Ia and BAO, we found that $\omega_{D E}<-1$, for all cases, which means our universe slightly biases towards phantom behavior while the values of $\Omega_{m_{0}}$, i.e. the present-day dark matter density, are very close to the desired value $\Omega_{m_{0}} \simeq 0.27$. Finally, using the best fit values of the model parameters in Table 2 , we have evolved the equation of state parameter, $\omega_{D E}$ for all cases I-IV in Figure 5 . The so-called phantom divide line crossing phenomenon has been clearly depicted in this figure. All in all, according to our analysis, a theory with a non-minimal coupling between the tachyon scalar field and the Gauss-Bonnet invariant is in agreement with cosmological observations and can be considered as a good candidate for dark energy.

\section{References}

[1] A.G. Riess, et al., Astron. J. 116, 1009 (1998); Astron. J. 117, 707 (1999).

[2] S. Perlmutter et al., Astrophys. J. 517, 565 (1999).

[3] Komatsu, E. et al., [WMAP Collaboration], Astrophys. J. Suppl. 192, 18 (2011).

[4] P. A. R. Ade et al., Astron. Astrophys. 594, A13 (2014)

[5] A. G. Riess et al., Astrophys. J. 699, 539 (2009).

[6] H. Lampeitl et al., Mon. Not. Roy. Astron. Soc. 401, 2331 (2009).

[7] M. Kowalski, et al., Astrophys. J. 686, 749 (2008).

[8] J. Martin, Comptes Rendus Physique 13, 566 (2012).

[9] S. Capozziello and M. De Laurentis, Phys. Rept. 509, 167 (2011).

[10] B. A. Bassett, S. Tsujikawa and D. Wands, Rev. Mod. Phys. 78, 537 (2006).

[11] E. J. Copeland, M. Sami and S. Tsujikawa, Int. J. Mod. Phys. D 15, 1753 (2006).

[12] Y. -F. Cai, E. N. Saridakis, M. R. Setare and J. -Q. Xia, Phys. Rept. 493, 1 (2010).

[13] C. Brans and R. Dicke, Phys. Rev. 124, 925 (1961).

[14] F.Bezrukov and M.Shaposhnikov, Phys.Lett. B 639, 703 (2008).

[15] V. Sahni and A. A. Starobinsky, Int. J. Mod. Phys. D 9, 373 (2000).

[16] V. Sahni and A. Starobinsky, Int. J. Mod. Phys. D 15, 2105 (2006); T. Padmanabhan, astro-ph/0603114 P. J. E. Peebles and B. Ratra, Rev. Mod. Phys. 75, 559 (2003); L. Perivolaropoulos, astro-ph/0601014; N. Straumann, arXiv:gr-qc/0311083 J. Frieman, arXiv:0904.1832, M. Sami, Lect. Notes Phys. 72, 219(2007); M. Sami, arXiv:0901.0756 ; K. Bamba, S. Capozziello, S. Nojiri and S. D. Odintsov, arXiv:1205.3421, S. Tsujikawa, arXiv:1004.1493.

[17] E. V. Linder, Rep. Prog. Phys. 71, 056901 (2008).

[18] R. R. Caldwell and M. Kamionkowski, arXiv:0903.0866.

[19] A. Silvestri and M. Trodden, arXiv:0904.0024.

[20] J. Frieman, M. Turner and D. Huterer, arXiv:0803.0982.

[21] G. W. Horndeski, Int. J. Theor. Phys. 10, 363 (1974). 
[22] C. Deffayet, X. Gao, D. A. Steer and G. Zahariade, Phys. Rev. D 84, 064039 (2011).

[23] A. De Felice, T. Kobayashi and S. Tsujikawa, Phys. Lett. B 706, 123 (2011).

[24] T. Kobayashi, M. Yamaguchi and J. Yokoyama, Prog. Theor. Phys. 126, 511 (2011).

[25] K. Kamada, T. Kobayashi, M. Yamaguchi and J. Yokoyama, Phys. Rev. D 83, 083515 (2011).

[26] T. Qiu and Y.T. Wang, JHEP 1504, 130 (2015).

[27] A. Maselli, H. O. Silva, M. Minamitsuji and E. Berti, Phys. Rev. D 92, 104049 (2015).

[28] R. Myrzakulov and L. Sebastiani, Astrophys. Space Sci. 361, 62 (2016).

[29] G. Cognola, et. al., arXiv:1601.00102 [gr-qc].

[30] A. Anabalon, A. Cisterna and J. Oliva, Phys. Rev. D 89, 084050 (2014).

[31] A. Cisterna and C. Erices, Phys. Rev. D 89, 084038 (2014).

[32] A. Cisterna, T. Delsate and M. Rinaldi, Phys. Rev. D 92, 044050 (2015).

[33] A. Cisterna, M. Cruz, T. Delsate and J. Saavedra, Phys. Rev. D 92, 104018 (2015).

[34] I. Antoniadis, J. Rizos and K. Tamvakis, Nucl. Phys. B 415, 497 (1994).

[35] M. Gasperini, M. Maggiore and G. Veneziano, Nucl. Phys. B 494, 315 (1997).

[36] R. Brustein and R. Madden, Phys. Rev. D 57, 712 (1998).

[37] S. Kawai, M. -a. Sakagami and J. Soda, Phys. Lett. B 437, 284 (1998).

[38] C. Cartier, E. J. Copeland and R. Madden, J. High Energy Phys. 01, 035 (2000).

[39] S. Nojiri, S. D. Odintsov and M. Sasaki, Phys. Rev. D 71, 123509 (2005).

[40] G. Calcagni, et. al., Nucl. Phys. B 752,404 (2006).

[41] S. Nojiri, S. D. Odintsov and M. Sami, Phys. Rev. D 74, 046004 (2006).

[42] S. Tsujikawa and M. Sami, J. Cosmol. Astropart. Phys. 0701, 006 (2007).

[43] S. Capozziello, A. N. Makarenko and S. D. Odintsov, Phys. Rev. D 87, 084037 (2013).

[44] T. Chiba, Phys. Rev. D 64, 103503 (2001).

[45] V. Faraoni, Int. J. Mod. Phys. D 11, 471 (2002).

[46] E. Elizalde, S. Nojiri and S.D. Odintsov, Phys. Rev. D 70, 043539 (2004).

[47] I. Quiros, et. al., Class. Quant. Grav. 27, 215021 (2010).

[48] M. Farhoudi, Gen. Rel. Grav. 41, 117 (2009).

[49] S. Nesseris, Phys. Rev. D 88, 123003 (2013).

[50] B. Spyros, S. Nesseris, and L. Perivolaropoulos, Phys. Rev. D 87, 123529 (2013).

[51] N. Suzuki et al., Astrophys. J. 746, 85 (2012).

[52] L. Perivolaropoulos, Phys. Rev. D. 71, 063503 (2005).

[53] W. J. Percival, et. al., Mon. Not. Roy. Astron. Soc. 401, 2148 (2010). 
[54] D. J. Eisenstein, et. al., [SDSS Collaboration], Astrophys. J. 633, 560 (2005).

[55] C. Blake, E. Kazin, et. al., Mon. Not. Roy. Astron. Soc. 418, 1707 (2011).

[56] G.-B. Zhao, et. al., Phys. Rev. D 72, 123515 (2005).

[57] R. R. Caldwell, M. Doran, Phys. Rev. D 72, 043527 (2005);

[58] B. Feng, X. Wang and X. Zhang, Phys. Lett. B 607, 35 (2005). 